\title{
Time Evolution of Fields in Strontium Ferrite Permanent Magnets
}

\author{
J. T. Volk*, B. C. Brown, G. W. Foster, W. Fowler, H. D. Glass, G. P. Jackson \\ Fermi National Accelerator Laboratory PO Box 500 Batavia, IL 60510
}

\section{Abstract}

Field strengths for strontium ferrite permanent magnets built for the Fermilab Recycler and $8 \mathrm{GeV}$ transfer line have been measured for the past 4 years. The ferrite magnetization exhibits a time dependence parameterized by $\mathrm{M} 1 / \mathrm{M}_{2}=-9 \times 10^{-4} \times \log \left(\mathrm{t}_{1} / \mathrm{t}_{2}\right)$ as determined from measurements of a gradient magnet. This parameterization has been checked against several other styles of permanent dipoles, and quadrupole magnets with good agreement.

\section{RGF-005}

The Fermilab Recycler ${ }^{1}$ is an antiproton storage ring made of 500 permanent magnets ${ }^{2}$. There are combined function gradient magnets ${ }^{3}$ and quadrupoles ${ }^{4}$ in this

\section{RGF005-1}

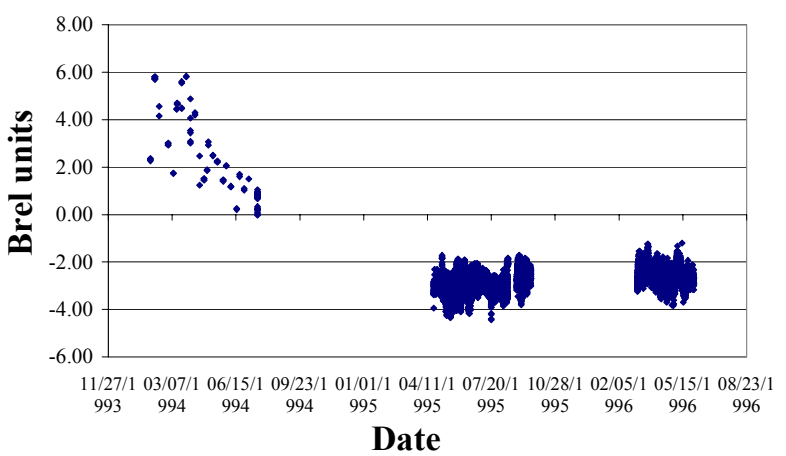

Figure 1: Date vs. field RGF005-1

machine. All magnets used machined steel poles to create the field shape and Grade 8 Strontium Ferrite to provide the magnetic field. A standard brick of $4 \times 6 \times 1$ inches was used. The bricks for the first production gradient magnet, RGF-005 were magnetized in November of 1997. The magnet was assembled in December of 1997. The magnetic field was adjusted by changing the amount of magnetic material and measured using a 4-meter Morgan coil. Since this was the first gradient magnet that met all the specifications for the Recycler ring this magnet was kept as a standard. It was measured once a week during the gradient production. After that it was

Manuscript received June 12, 2001

*Volk@fnal.gov

Work supported by the U.S. Department of Energy under contract No. DE-AC02-76CH03000. measured from time to time for periods of several days or weeks. Since the field is produced entirely by the ferrite, the measured field strength is proportional to the ferrite magnetization. A plot of date of measurement versus the relative field strength $\left(\right.$ brel) ${ }^{4}$ shows logarithmic time (Figure 1) dependence $^{5}$. A linear regression fitting log (day) since magnetization versus brel yields

$$
\mathrm{M}_{2} / \mathrm{M}_{1}=-9 \times 10^{-4} * \log \left(\mathrm{t}_{2} / \mathrm{t}_{1}\right)
$$

Where $\mathrm{M}$ is the magnetization and $\mathrm{t}$ is the time in days. This parameterization can be used to determine the expected time dependence of other magnets built for the recycler ring.

\section{RQRA}

There were 32 20-inch long quadrupoles built for a phase trombone but not installed. The first four RQRA magnets were built in October 1997, the remaining 28 were built in October

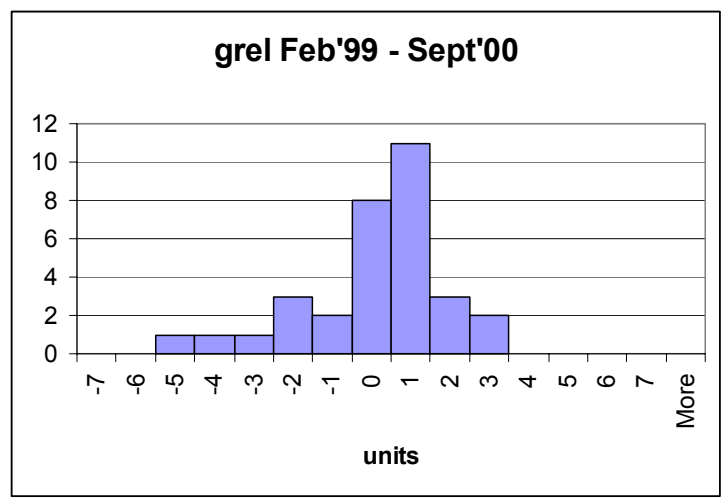

Figure 2: Change in RQRA gradient

1998. The strength of all 32 magnets was adjusted in February of 1998 and the magnets were put into storage until August of 2000. The relative gradient (grel) were measured for each magnet and the difference between the February 1999 and the September 2000 data is plotted in figure 2. The grel is expressed in terms of units, where 1 unit is equal to 1 x $10^{-4}$ the histograms is centered at 0 with a small negative tail. The expected change in grel is -5.5 units. 


\section{PVB}

For the $8 \mathrm{GeV}$ transfer line between the Booster and the Main Injector there were five vertical bend magnets (PVB) built. Due to aperture restrictions these magnets were removed from the beamline. In the summer of 2000 three of these magnets were sent to Jefferson lab for use there. Before shipping, these magnets were measured. The relative dipole field (brel) were measured to be -15, 25 , and -27 units. There was a total of 1362 days between magnetization of the bricks and these measurements. The predicted brel change was -28 units, this is in good agreement with the parameterization.

\section{RQME}

As part of the MI-30 upgrade 8 20-inch quads (RQME) were removed from the Recycler Ring

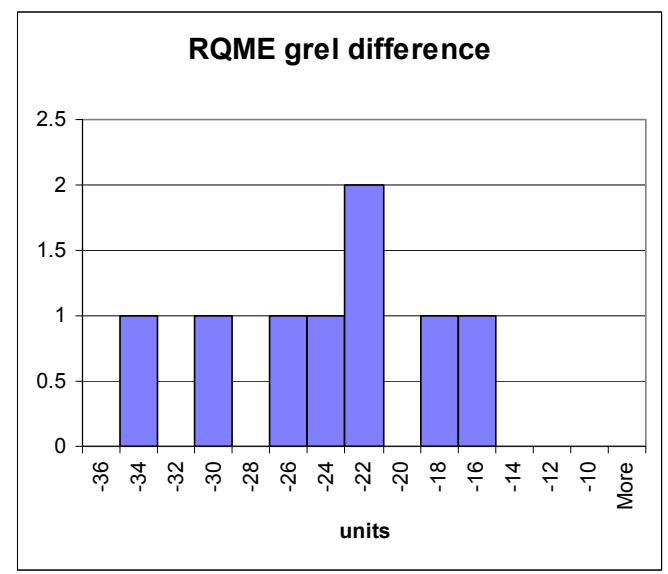

Figure 3: RQME change in gradient

(tunnel) and the gradient changed. Each magnet was first measured to assess the change in field. These bricks were magnetized on July of 1998. They were re-measured 860 days after that. The expected change in grel is -26 units The average is $-25 \pm 5$ units

\section{RQES}

For the high beta region of the recycler 12 large aperture 1-meter long quads were built. The magnets were built in September 1998 and given a final tune up in December of 1998. Due to the strict requirements on the gradients the strength of these magnets were all adjusted at $32{ }^{\circ} \mathrm{C}$. This was accomplished by placing the magnets in a temperature controlled box and stabilizing the magnet temperature at $32{ }^{\circ} \mathrm{C}$ for 24 hours before adjusting the strength. When the magnets were installed in the tunnel they were covered with heating blankets and maintained at $32{ }^{\circ} \mathrm{C}$. There was one magnet that was not installed in the tunnel but kept in storage as a standard calibration magnet. For the ten magnets installed in the tunnel the change in grel was measured to be $-30 \pm 5$ units (Figure 4). For the standard magnet the change was 5 units. The predicted change was -8 units. The parameterization agrees well for the standard magnet. The extra 25 units of change for the magnets which had been installed in the tunnel is due to keeping the magnets at an elevated temperature for 2 years. The theory was presented in a paper given in the 1997 Particle Accelerator Conference ${ }^{7}$ The rate of change $\mathrm{N}$ due to thermal

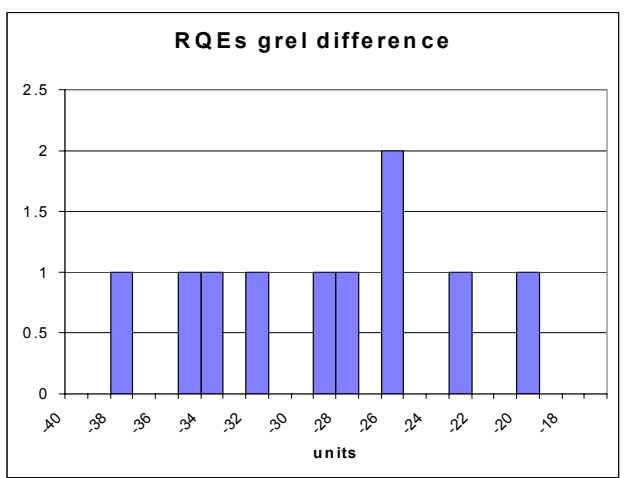

Figure 4: RQE Grel Difference

activation can be expressed as:

$$
d N / d E=-C f(E, t) e^{-E / k T} d E
$$

The $\mathrm{e}^{-\mathrm{E} / \mathrm{kT}}$ term explains the change in the grel for these magnets.

\section{CONCLUSION}

The time evolution for strontium ferrite magnets has been parameterized from measurements conducted on a single magnet. This parameterization has been tested against several other magnets of different styles and found to predict the observed decrease in strength for magnets held at tunnel temperature. At elevated temperature the magnetization decreases more quickly. Changing the orbit by occasional realignment of the magnets can compensate the change in strength of the recycler magnets.

\section{REFERENCES}

[1]. Stephen D Holmes "Status of the Fermilab Main Injector and Recycler" 1997 Particle Accelerator Conference Conf 97-46

[2] H.D Glass, "Permanent magnets for beamlines and the recycler ring at Fermilab", 17th International Conference on High-Energy Accelerators (HEACC 98), Dubna, Russia, 7-12 Sep 1998 Pages 283-287 
[3] B. C. Brown et al., "Hybrid Permanent Magnet Gradient Dipoles for the Recycler Ring at Fermilab", Proceedings of 15th International Conference on Magnet Technology, 161, 1998.

[4]. Brel and grel are defined as: (measured - design) /design

[5]. B. C. Brown et al., "Time Dependence and Temperature Stability of the Permanent Magnets for the Fermilab Antiproton Recycler Ring ", IEEE Trans. on Applied Superconductivity 10, 879,2000.

[6] B. C. Brown et al., "Hybrid Permanent Magnet Quadrupoles for the Recycler Ring at Fermilab", Proceedings of 15th International Conference on Magnet Technology, 183, 1998.7) H.D. Glass et. al., "Stability Tests of Permanent Magnets Built with Strontium Ferrite", Proceedings of the 1997 Particle Accelerator Conference, 3260, 1998. 